\title{
AEROGEL INSULATION SYSTEMS \\ FOR SPACE LAUNCH APPLICATIONS
}

\author{
J.E. Fesmire \\ NASA Kennedy Space Center, YA-C2-T \\ Kennedy Space Center, FL, 32899, USA \\ James.E.Fesmire@nasa.gov \\ phone: (321) 867-7557 \\ fax: (321) 867-4446
}

\begin{abstract}
New developments in materials science in the areas of solution gelation processes and nanotechnology have led to the recent commercial production of aerogels. Concurrent with these advancements has been the development of new approaches to cryogenic thermal insulation systems. For example, thermal and physical characterizations of aerogel beads under cryogenic-vacuum conditions have been performed at the Cryogenics Test Laboratory of the NASA Kennedy Space Center. Aerogel-based insulation system demonstrations have also been conducted to improve performance for space launch applications. Subscale cryopumping experiments show the thermal insulating ability of these fully breathable nanoporous materials. For a properly executed thermal insulation system, these breathable aerogel systems are shown to not cryopump beyond the initial cooldown and thermal stabilization phase. New applications are being developed to augment the thermal protection systems of space launch vehicles, including the Space Shuttle External Tank. These applications include a cold-boundary temperature of $90 \mathrm{~K}$ with an ambient air environment in which both weather and flight aerodynamics


are important considerations. Another application is a nitrogen-purged environment with a cold-boundary temperature of $20 \mathrm{~K}$ where both initial cooldown and launch ascent profiles must be considered. Experimental results and considerations for these flight system applications are discussed.

\section{INTRODUCTION}

Much has been made in recent years of the application of aerogels for thermal insulation. The roots of aerogel materials extend back to the 1930 s when Kistler invented a process for making a porous inorganic silica product [1]. About 50 years later modern aerogels were developed, coinciding with the advent of solution-gelation (sol-gel) chemical processing methods. These new aerogels are distinguished by their very fine pore sizes (from about 1 to 20 nanometers). The very small pores are synonymous with very high surface areas (such as from 800 to 1,100 meter $^{2}$ per gram) of the internal structures. These new aerogels can be made to have ultra-low densities. The modern sol-gel processing techniques behind these materials allows for tailoring the molecules for a given application. The modern aerogels are often characterized as having an ultralow density in the monolithic (solid) form, but this is not an essential distinguishing feature. Application of aerogels for thermal insulation were studied by Hrubesh [2], Smith et al. [3], and others.

In the last five years large-scale commercial production of the modern, nanaoporous aerogel materials has been achieved. The financial motivation for this business development was due, in large part, to the potential applications in thermal insulation. For example, products now available include aerogel beads manufactured by Cabot Corporation [4] and aerogel composite blankets manufactured by Aspen Aerogels, Inc. [5]. Because they are fully breathable and hydrophobic, these materials are ideal candidates for thermal insulators in a number of space launch applications. 


\section{THE INSULATION PROBLEM ON SPACE LAUNCH VEHICLES}

As a general rule, cryogenic thermal insulation systems should be either completely sealed or fully breathable with the surrounding environment. Systems that are partially or imperfectly sealed can lead to reliability or safety problems because the residual matter within is not fully known. One major concern with space launch vehicles is the migration of air or water molecules from the warm side to the cold side during the transient cooldown processes of the cryogenic propellant loading operations. Dense air, ice, water, and (in the case of liquid-hydrogen tanks or pipes), liquefied air, can accumulate within the insulation materials because of this mass transport effect (commonly referred to as cryopumping). These accumulations can degrade vehicle performance in several ways, including increased heat transfer through the thermal insulation, increased lift-off weight, and the potential for damaging ice debris.

The problem of accumulated matter within the insulation is further compounded when the launch vehicle must ascend from the ambient pressure environment of the launch site to the high-vacuum environment of space. The molecules trapped inside the insulation materials must then escape to the lower pressure side without damaging the thermal protection system, adjacent hardware, or flight surfaces. The rapid heating effects due to the aerodynamics at high mach numbers only serve to further worsen the problem.

\section{CRYOPUMPING EFFECTS IN AEROGELS}

Cryopumping is strictly and simply defined as the production of a vacuum using low temperatures. The four basic mechanisms of cryopumping are cryocondensation, cryosorption, cryotrapping, and cryogettering. The mechanisms cryocondensation and cryosorption, both brought about by van der Waals' forces, are most relevant to the aerogels used on liquid hydrogen or liquid oxygen systems [6]. When gas molecules impinge a low-temperature surface they lose much kinetic energy and can become attached to that surface. Eventually an equilibrium point will be reached where the rates of particles impinging and departing the surface are equal. 
Applied to a thickness of porous insulation material, this point would be one of an infinite number of isotherms. Cryopumping effectively ceases for a well-insulated system after initial cooldown is achieved.

Aerogels are high-performance thermal insulators in ambient or partial vacuum environments because of their extremely small pore sizes which greatly impede the communication of gas molecules by thermal conduction. A properly designed thermal insulation system based on aerogel material will also minimize all interstitial spaces within the system, thereby lowering the heat transfer caused by gas convection. During the transient process of cooldown from ambient to cryogenic temperature, the gases within the system will, to some degree, tend to migrate to the cold side and condense or freeze. But these cryopumping effects are lessened because of the very fine pore size and extraordinarily low thermal conductivity of the aerogel. Furthermore, because the aerogels are such good thermal insulators, the isotherms between the warm side and the cold side are more readily established. The result is that cryopumping essentially stops after the transient cooldown period. The near steady state condition typical of ambient pressure systems must be maintained for approximately 8 hours to be sufficient for the launch vehicle propellant conditioning and hold period.

The main work of the cryogenic insulation system is done at lift-off. The transient warmup phase, starting at launch and continuing during the several minutes of ascent into the vacuum of space, allows the additional trapped molecules to readily escape through the porous aerogel into the lower pressure environment with no harm to the vehicle thermal protection systems.

\section{AEROGEL THERMAL PERFORMANCE CHARACTERIZATION}

Looking in closer detail at the physical characteristics of aerogels shows the possibilities for new thermal insulation systems for both low-temperature and high-temperature uses. Figure 1 presents the variation of the apparent thermal conductivity with the cold vacuum pres- 
sure for an aerogel blanket (two layers, $16 \mathrm{~mm}$ total thickness) and an aerogel bead ( $1 \mathrm{~mm}$ nominal diameter, $25 \mathrm{~mm}$ total thickness) material. Comparisons with three commonly used cryogenic insulation materials, polyurethane spray foam, perlite powder, and multilayer insulation (MLD), are also given. These experimental data were obtained using a liquid nitrogen boiloff cryostat in a cylindrical configuration. Details on this new standardized equipment and method are given in the literature $[7,8]$.

The foam material, type BX-250, was tested at a thickness of $51 \mathrm{~mm}$ and with a measured density of $38 \mathrm{~g} / \mathrm{cm}^{3}$. The thermal performance data for the aerogel beads and perlite powder has been previously reported [9]. Details on the 60-layer MLI system shown here have also been previously reported [10].

Both aerogel materials are silica-based, fully breathable, and treated to be hydrophobic. The aerogel blanket was composed of two layers, $8 \mathrm{~mm}$ thickness each, with an overall bulk density of $51 \mathrm{~kg} / \mathrm{m}^{3}$. Details on aerogel composite blanket materials are given in the papers by Fesmire and Ryu [11,12]. The aerogels beads are 90 percent porous with a mean pore diameter of $20 \mathrm{~nm}$ and an internal surface area around $800 \mathrm{~m}^{2} / \mathrm{g}$. With a bead density of $140 \mathrm{~kg} / \mathrm{m}^{3}$ and a typical bulk density of $80 \mathrm{~kg} / \mathrm{m}^{3}$, the interstitial space is approximately 60 percent. The beads have a fully elastic compression capability of well over 50 percent with no significant damage. The yield strength of an individual aerogel particle allows for volumetric compression of up to 30 percent with full reversible behavior [13]. This packing feature supports the application of this material as a thermal insulator for ambient pressure environments. Further information on the aerogel bead materials can be found in the paper by Ackerman and Rouanet [14].'

To investigate the different mass-transport effects through the nanoporous aerogel materials and show their relevance to low-temperature thermal insulation performance, a number of cryopumping experiments were devised. Two of these experiments using aerogel beads are described in the following sections. 


\section{CRYOPUMPING EXPERIMENT USING BULK-FILL CRYOSTAT}

A research test cryostat was filled to a level of approximately $200 \mathrm{~mm}$ above the top of the liquid nitrogen cold mass tank as depicted in Figure 2. The vacuum chamber was stabilized with room air at ambient conditions to simulate the launch vehicle environment. The closed mass system was then cooled from approximately $293 \mathrm{~K}$ to $77 \mathrm{~K}$ while the drop in pressure from $101 \mathrm{kPa}$ (760 torr) was monitored. The system pressure was lowest at approximately $80 \mathrm{kPa}$ (600 torr) in less than 1 hour from the start of cool-down. Pressure stabilized about 2 hours after liquid nitrogen filling was completed (Figure 3). The feedthrough temperature trace during cool-down and warm-up explains the temporary increase in pressure between the 2-hour and 3-hour marks. It is interesting to note that this same base pressure was reached upon filling, indicating that the cryopumping occurs only in the close proximity to the cold mass surface and only at the beginning of the process.

\section{CRYOPUMPING EXPERIMENT USING COLD-COLUMN APPARATUS}

A cold-column apparatus was devised to simulate the internal launch vehicle environment of a large purged cavity, of gaseous nitrogen at $293 \mathrm{~K}$ in contact with a cold surface at $20 \mathrm{~K}$. The thermal similarity between the flight vehicle case and the experimental case is depicted in Figure 4 showing the liquid-hydrogen tank surface, the thin layer of solid nitrogen, and the thin layer of liquid nitrogen. A 150 -mm-tall column of aerogel beads was then interposed with temperature sensors at 25-mm intervals (see Figure 4). The aerogel was held within the G-10 tube column using a fiberglass cloth at the bottom. The top surface was open to room air. The column was set into a pair of dewar flasks and temperatures were monitored at the different heights within the column. The liquid-nitrogen level was maintained manually by transfer from a third dewar flask. The bottom of the column was inserted $12.5 \mathrm{~mm}$ below the surface of the liquid nitrogen. 
Several long-duration runs of the experiment were made with similar results. The aerogel-bead-filled column temperatures as a function of time are presented in Figure 6. Isotherms formed in approximately 3 hours from the start of cooldown (initial immersion of the lower end of the column). The results show that liquid quickly filled to some level between $0 \mathrm{~mm}$ and $25 . \mathrm{mm}$, and that there was no appreciable change even after the 12-hour runtime. The isotherms at $50 \mathrm{~mm}, 75 \mathrm{~mm}$, and so forth were as expected according to previous thermal conductivity measurements of the aerogel beads [99]. In this case, cryopumping within the entire column ceased after about 3 hours, at which time state-state conditions were observed and kept for another 9 hours. Note that the periodic variations in temperature are attributed to the manual method of liquid-nitrogen level control. The slow warm-up process is seen from hours 12 to 18 as the sequestered nitrogen molecules are liberated from the fine pores of the aerogel.

\section{FLIGHT VEHICLE APPLICATION: LIQUD-OXYGEN FEEDLINE BELLOWS}

A liquid-oxygen feedline with exposed metal bellows is a good candidate for an aerogel-based insulation system because the bellows must be free to move without collecting any ice. Ice is a primary hazard for the vehicle surfaces and could also impede the mechanical articulation of the bellows assembly. A photo of the feedline area on the External Tank of the Space Shuttle is shown in Figure 7. The aerogel insulation system has undergone development testing as seen in Figure 8.

This testing demonstrated the aerogel-based system to be fully effective in eliminating frost or ice. Another key aspect demonstrated was the hydrophobic nature of the aerogel such that no ice formations occurred within the internal cold cavities during the 8 to 10 hours of simulated ground-hold conditions. Cryopumping effects were stopped upon reaching thermal stabilization through the thickness of the insulation materials. The aerogel beads, being nanoporous, tightly packed, and hydrophobic, further inhibited the movement of moisture or air into the cavity. 


\section{FLIGHT VEHICLE APPLICATION: LIQUID HYDROGEN TANK DOME}

A liquid-hydrogen tank dome with an exposed periphery of cold metal is a good candidate for an aerogel-based insulation system because the steady accumulation of liquefied nitrogen from the large purged cavity above the dome will lead to additional flight mass and could cause collateral damaging effects to the flight thermal protection system on the vehicle's outside surface. Figure 7 shows the upper portion of the liquid-hydrogen tank (the dome to flange area) on the Space Shuttle. A photo of the one-tenth-scale test article for demonstrating another aerogel-based insulation system is given in Figure 9.

The unit is sprayed with foam insulation except for one narrow area about the circumference of the upper dome of the tank. Liquid helium is used to obtain the necessary cold boundary temperature while the pressure inside the nitrogen-purged cavity above the dome is monitored. In this way the cryopumping effects as a function of propellant loading time can be determined. The objective of this demonstration testing is to show that the aerogel-based insulation system stops liquefaction of the nitrogen purge gas after cooldown is complete, as shown in the research experiment with the cold-column apparatus.

\section{CONCLUSIONS}

Aerogels provide new possibilities for thermal insulation systems for both lowtemperature and high-temperature applications on space launch vehicles. Experimental research testing shows that the cryopumping effects through the aerogel-based material systems, even though they are open-cell porous molecular networks, effectively cease upon completion of cooldown. Further testing has shown these materials to be fully hydrophobic with no tendency to produce ice-laden debris that could be hazardous to a vehicle during flight. Current system demonstration tests at the Cryogenics Test Laboratory confirm problem-solving applications for existing space launch vehicles including the Space.Shuttle. All launch vehicles must have thermal protection systems that function reliably in both ambient pressure and high- 
vacuum environments. Future vehicles that must be robust or reusable will likely be enabled by the aerogel technologies now being developed.

\section{ACKNOWLEDGEMENTS}

The author thanks Stan Augustynowicz for performing the thermal characterization and analysis of the materials. The author thanks Charles Stevenson and Trent Smith for their valuable insight and support throughout this research work. 


\section{REFERENCES}

[1]. Kistler, S.S., The Relation Between Heat Conductivity and Structure in Silica Aerogel, Journal of Physical Chemistry, 39, 79, 1935.

[2]. Hrubesh, Lawrence W, Aerogel Applications, Journal of Non-crystalline Solids. 225, 335, 1998.

[3]. Smith, Douglas M, et al., Aerogel-based thermal insulation, Journal of Non-crystalline Solids, 225, 254, 1998.

[4]. Cabot Corp., http://w1.cabot-corp.com/controller.jsp?N=23+4294967102+3153\&entry-product

[5]. Aspen Aerogels, Inc., http://www.aerogel.com/products.htm

[6]. Haefer, R. A., Cryopumping, Theory and Practice, Clarendon Press, Oxford, 1989, pp. 54-55.

[7]. Fesmire, J.E., Augustynowicz, S.D., Heckle, K.W., and Scholtens, B.N., Equipment and Methods for Cryogenic Thermal Insulation Testing, Advances in Cryogenic Engineering, American Institute of Physics, New York, 2004, Vol. 49, pp. 579-586.

[8]. Fesmire, J.E. and Augustynowicz, Methods of Testing Thermal Insulation and Associated Test Apparatus, US Patent 6,742,926 June 1, 2004.

[9]. Fesmire, J.E., Augustynowicz, S.D., and Rouanet, S., Aerogel Beads as Cryogenic Thermal Insulation System, Advances in Cryogenic Engineering, American Institute of Physics, New York, 2002, Vol. 47, pp. 1541-1548.

[10].Fesmire, J.E., Augustynowicz, S.D. and Demko, J.A., Thermal Insulation Performance of Flexible Piping for Use in HTS Power Cables, Advances in Cryogenic Engineering, American Institute of Physics, New York, 2002, Vol. 47, pp. 1525-1532.

[11].Fesmire, J.E., Rouanet, S., and Ryu, J., Aerogel-Based Cryogenic Superinsulation, Advances in Cryogenic Engineering, Plenum Press, New York, 1998, Vol. 44, pp. 219-226.

[12].Fesmire, J.E. and Ryu, J., Improved Aerogel-Based Thermal Insulation Systems, NASA Tech Briefs, June 1999, pg. 56.

[13]. Woignier, $T$., et al., Plastic densification in silica aerogels, European Physical Journal Applied Physics, 6, 1999, pp. 267-271.

[14]. Ackerman, W.C., Vlachos, M., Rouanet, S., Fruendt, J., Use of surface treated aerogels derived from various silica precursors in translucent insulation panels, Journal of Non-crystalline Solids, 2001, Vol.285, No. 1-3, pp. $264-271$. 
FIGURE 1. Apparent thermal conductivity as a function of cold vacuum pressure for aerogels compared to different insulation materials. $78 \mathrm{~K}$ and $293 \mathrm{~K}$ boundary temperatures; residual gas nitrogen. 


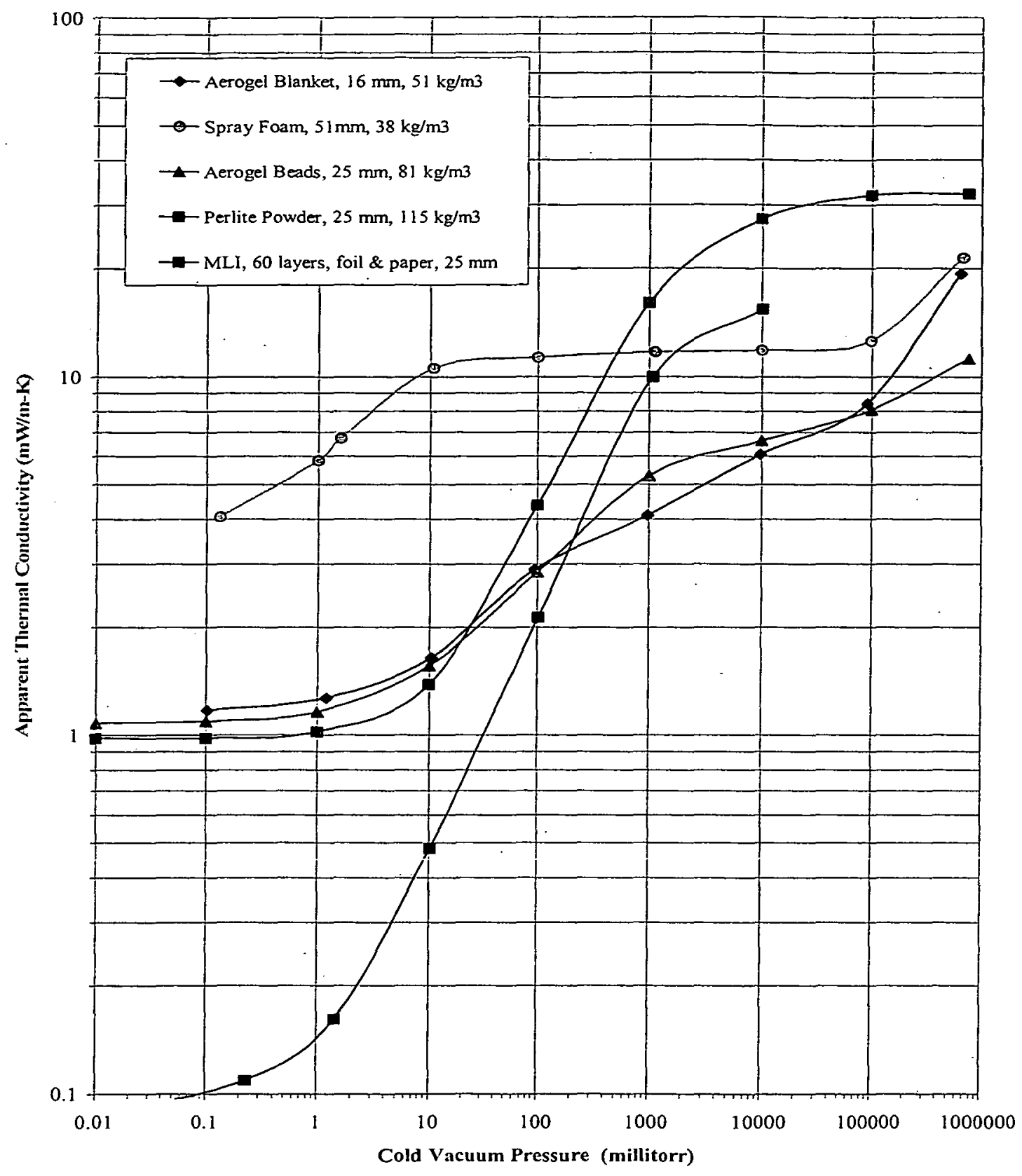


FIGURE 2. Cryopumping experimental test apparatus using cryostat loaded with bulk-fill aerogel beads. 


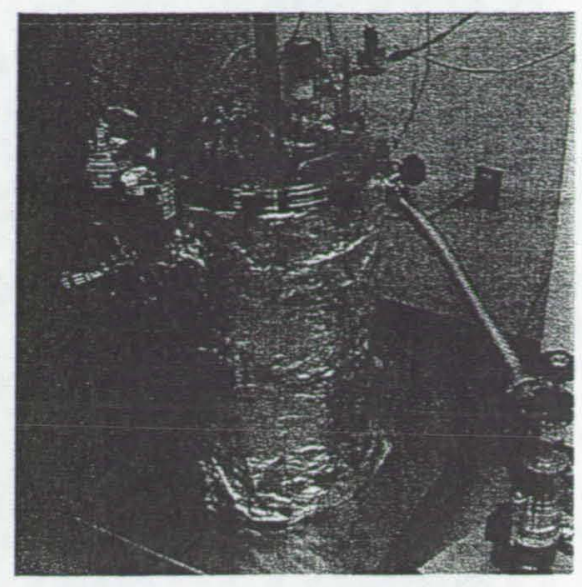

\begin{tabular}{|c|c|}
\hline \multicolumn{2}{|c|}{ Surtace Tomperature Meanurement } \\
\hline Sensor & Location \\
\hline T1, T3 & Cold-Boundary Temperature (CBT) \\
T2 & Tod Cold-Mass \\
T4. T5 & Warm-Boundary Temperature WBT) \\
T6 & Liquid Nitrogen Feedhrough \\
VC1. VC2, VC3 & Vacuum Chamber Exterior \\
VC4. VCS, VC6 & Heater Terrperature \\
\hline
\end{tabular}

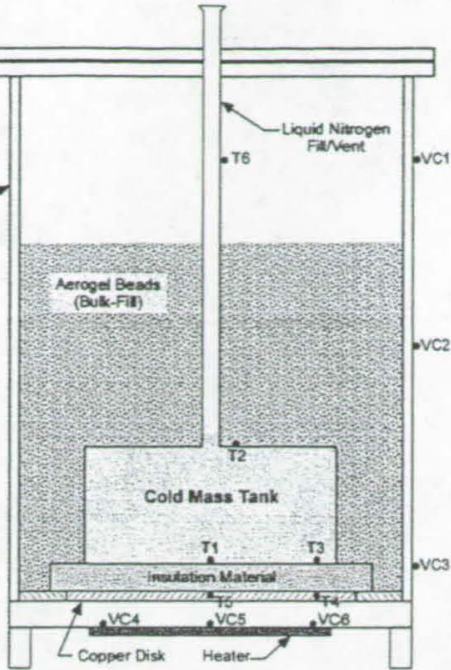


FIGURE 3. Cryopumping experiment using aerogel beads. Residual gas nitrogen. Closed mass system shows stabilized pressure approximately 2 hours after liquid nitrogen filling is complete. 


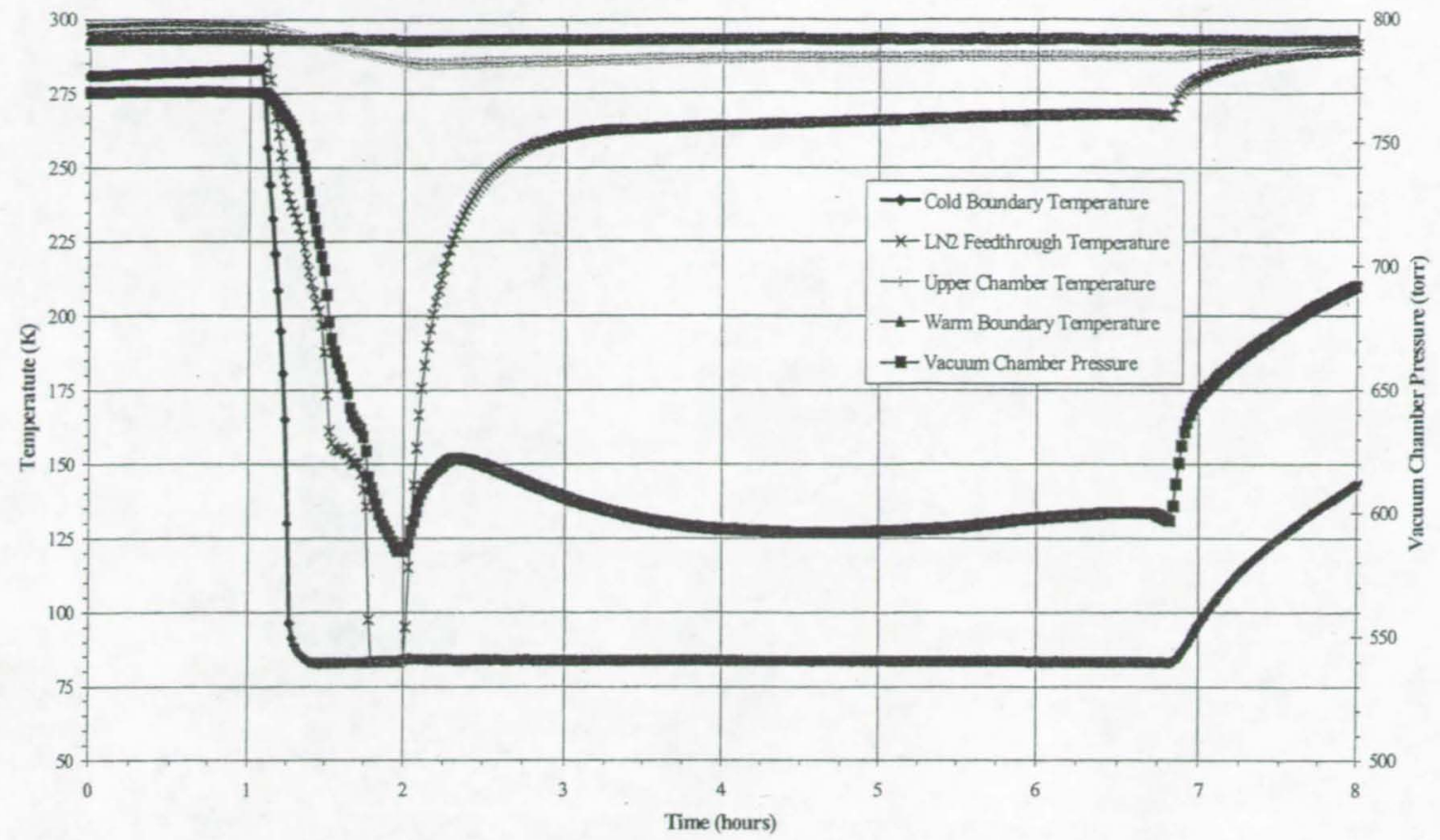


FIGURE 4. Thermal similarity between the flight vehicle and the experimental case of pooled liquid nitrogen within a large purged cavity with a cold boundary temperature of $20 \mathrm{~K}$. 
Flight Vehicle Case

$\underline{\text { Proof-of-Concept Test Case }}$

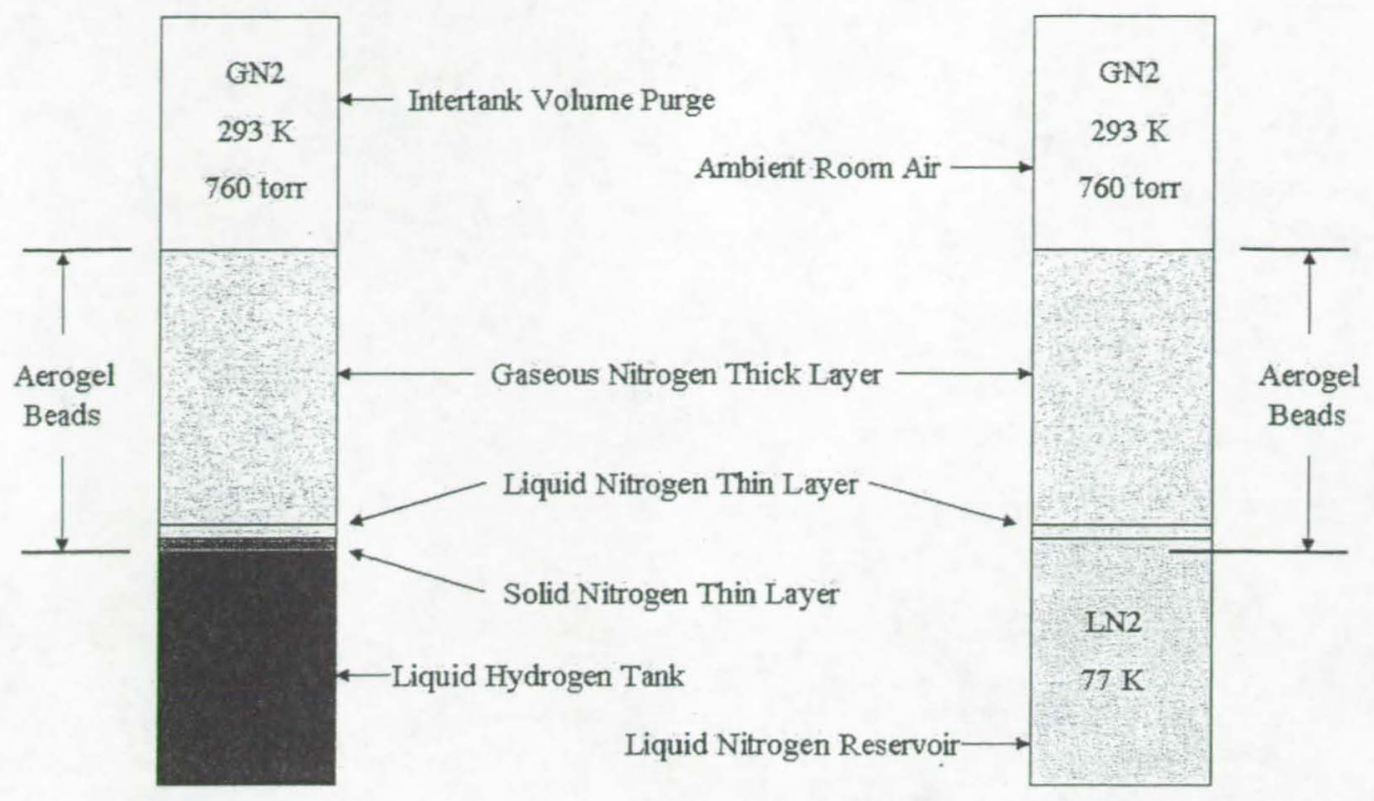


FIGURE 5. Cryopumping experimental apparatus with liquid nitrogen reservoir and cold column: (L) Postion of thermocouple sensors inside column, $(R)$ overall apparatus of aerogel-filled column set into dewar flask. 

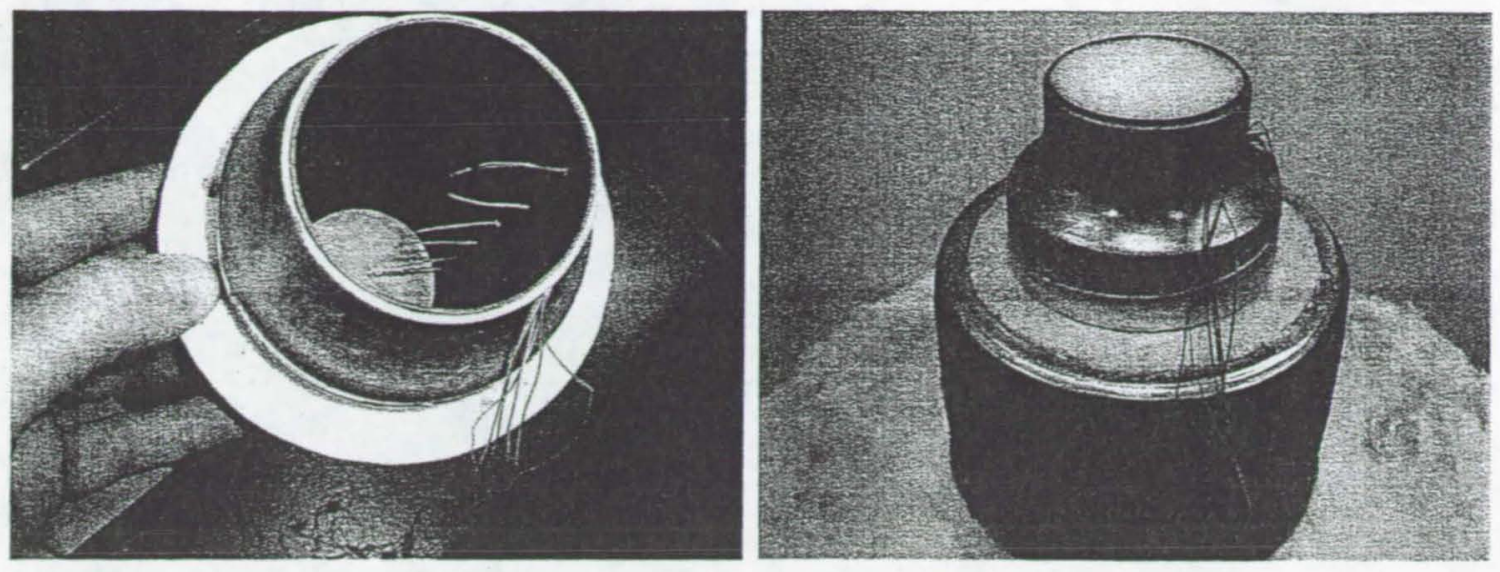
FIGURE 6. Cryopumping with liquid-nitrogen reservoir. Isotherms in aerogel beads column form after approximately 3 hours. Room air on warm side. 


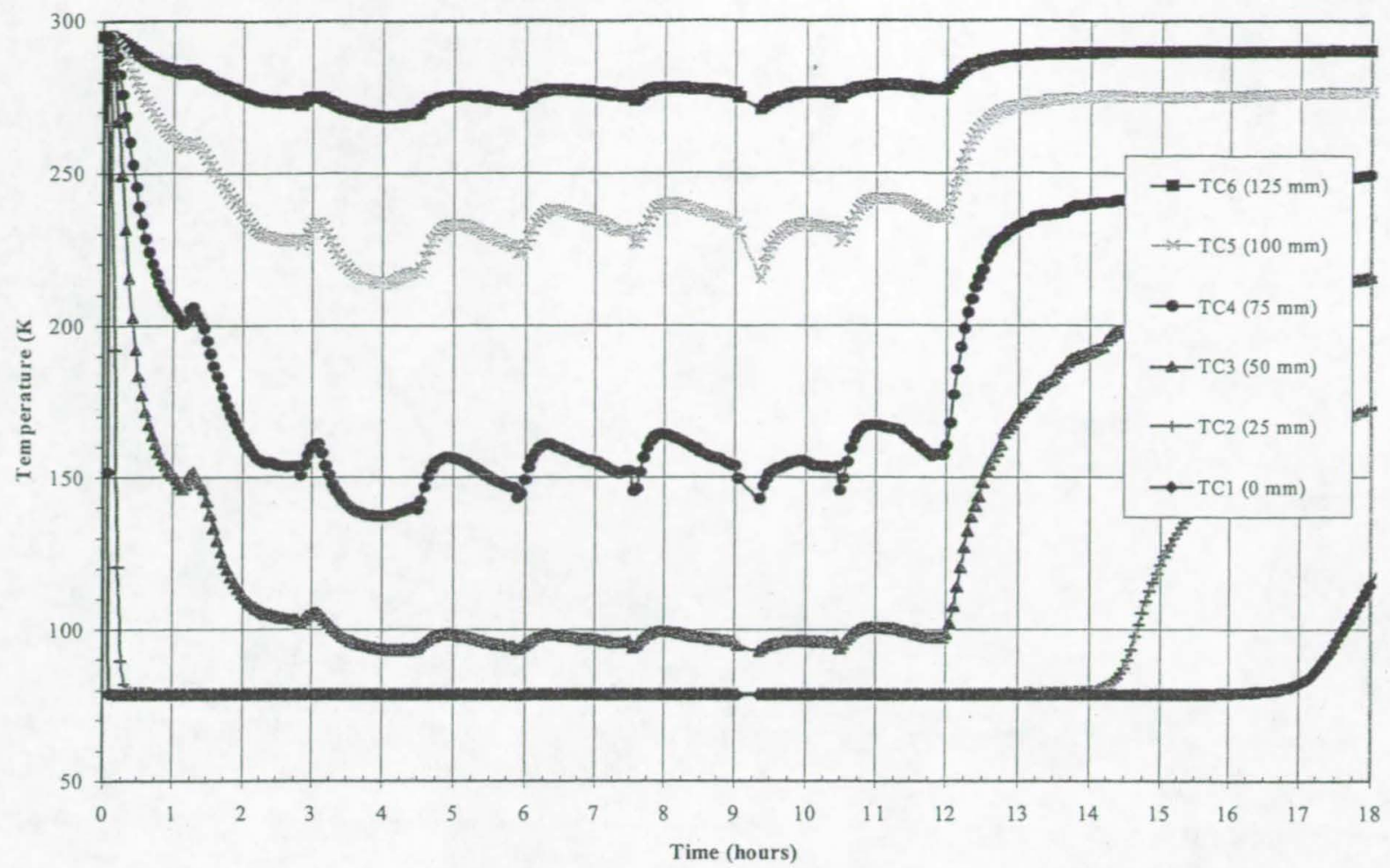


FIGURE 7. Views of the Space Shuttle External Tank. The right photo shows a portion of the liquid-oxygen feedline and upper portion of the liquid-hydrogen tank. 

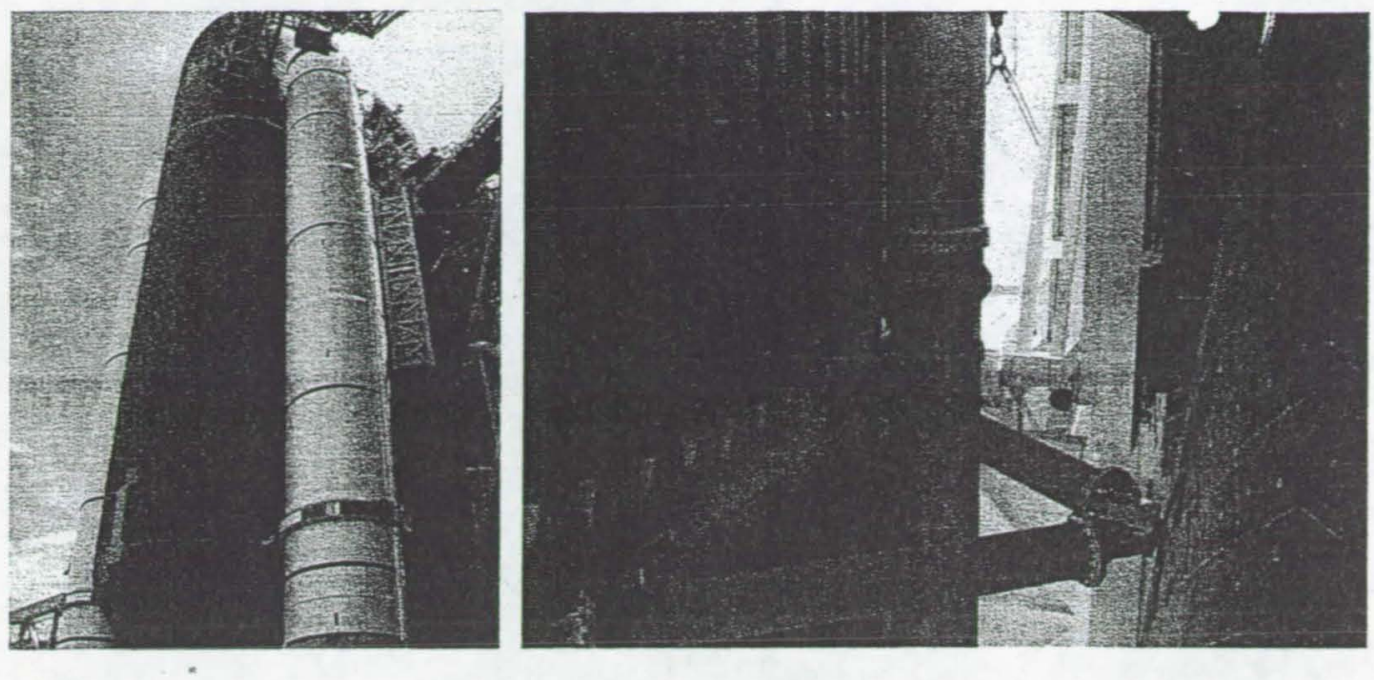
FIGURE 8. Liquid-oxygen feedline bellows shown schematically on the left. The right photo show's full-scale thermal-mechnical testing in progress of the aerogel-based thermal insulation system. 

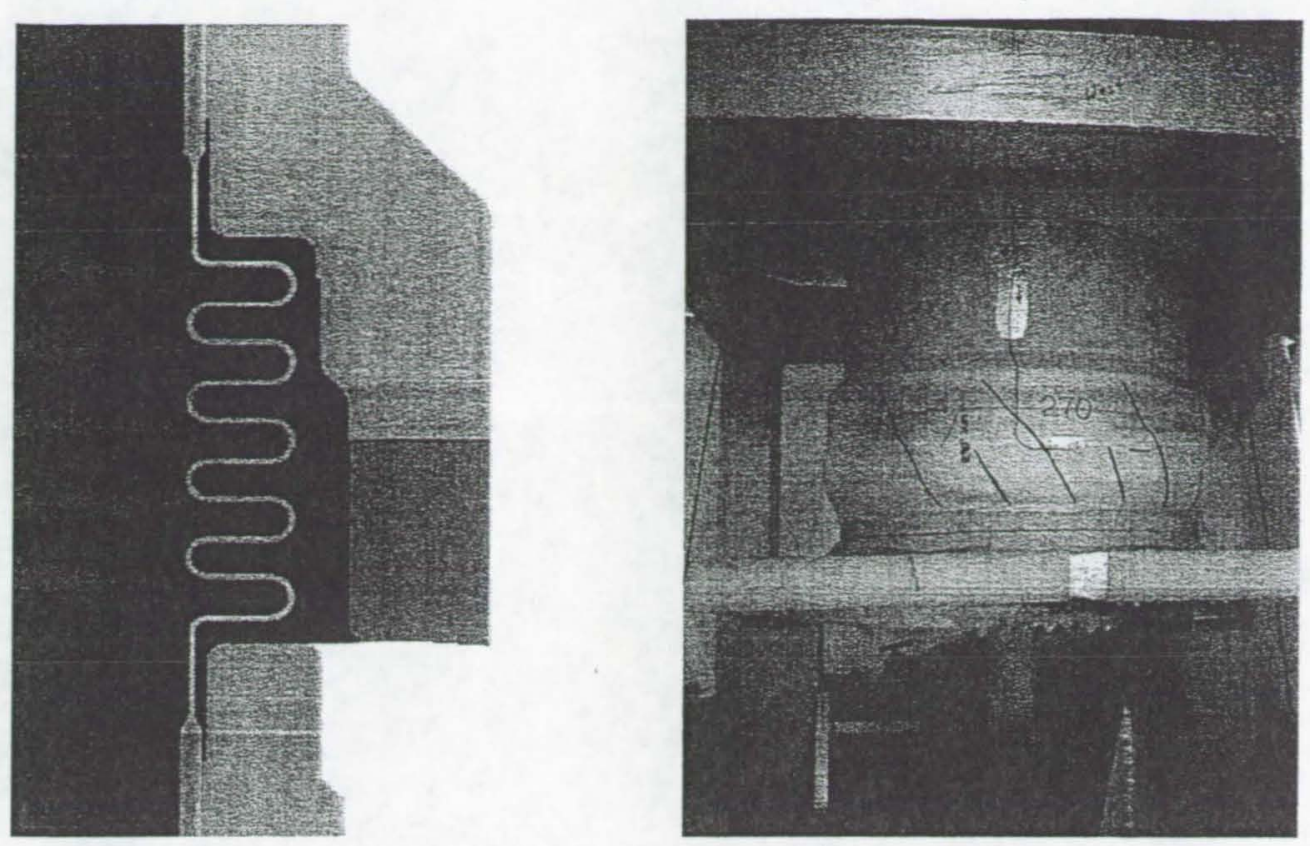
FIGURE 9. A demonstration unit for a liquid-hydrogen tank application is shown being prepared for evaluating another aerogel-based insulation system. 

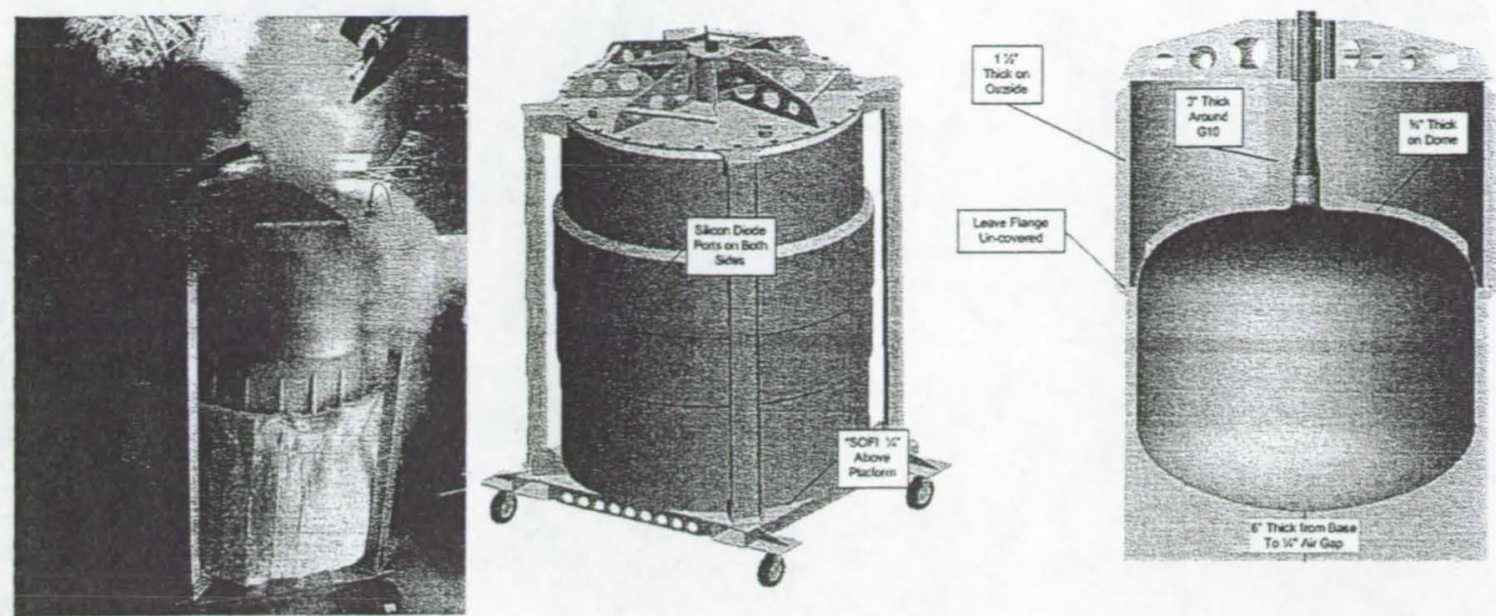